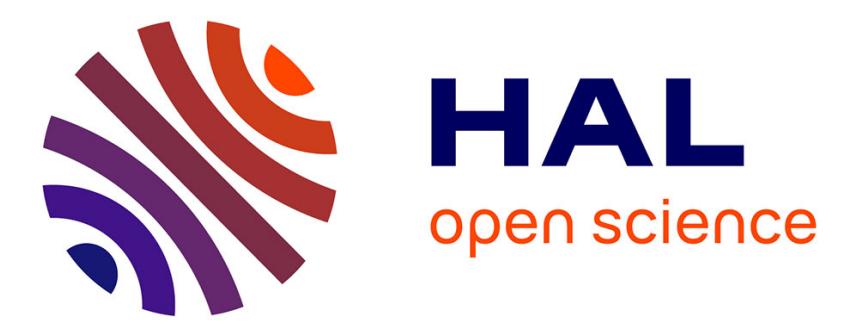

\title{
A Constraint-based Language for Declarative Pattern Discovery
}

Jean-Philippe Metivier, Patrice Boizumault, Bruno Crémilleux, Medhi Khiari, Samir Loudni

\section{- To cite this version:}

Jean-Philippe Metivier, Patrice Boizumault, Bruno Crémilleux, Medhi Khiari, Samir Loudni. A Constraint-based Language for Declarative Pattern Discovery. Data Mining Workshops (ICDMW), 2011 IEEE 11th International Conference on, Dec 2011, vancouver, Canada. p.1112-1119, 10.1109/ICDMW.2011.11. hal-01017223

\section{HAL Id: hal-01017223 \\ https://hal.science/hal-01017223}

Submitted on 15 Jul 2014

HAL is a multi-disciplinary open access archive for the deposit and dissemination of scientific research documents, whether they are published or not. The documents may come from teaching and research institutions in France or abroad, or from public or private research centers.
L'archive ouverte pluridisciplinaire HAL, est destinée au dépôt et à la diffusion de documents scientifiques de niveau recherche, publiés ou non, émanant des établissements d'enseignement et de recherche français ou étrangers, des laboratoires publics ou privés. 


\title{
A Constraint-based Language for Declarative Pattern Discovery
}

\author{
Jean-Philippe Métivier ${ }^{1,2}$, Patrice Boizumault ${ }^{1,2}$, Bruno Crémilleux ${ }^{1,2}$, Mehdi Khiari ${ }^{1,2}$, Samir Loudni ${ }^{1,2}$ \\ ${ }^{1}$ Université of Caen Basse-Normandie, GREYC, 14032 Caen - France \\ ${ }^{2}$ CNRS UMR 6072, GREYC, 14032 Caen - France \\ \{firstname.lastname\}@unicaen.fr
}

\begin{abstract}
Discovering pattern sets or global patterns is an attractive issue from the pattern mining community in order to provide useful information. By combining local patterns satisfying a joint meaning, this approach produces patterns of higher level and thus more useful for the end-user than the usual local patterns. In parallel, recent works investigating relationships between data mining and constraint programming (CP) show that the CP paradigm is a powerful framework to model and mine patterns in a declarative and generic way. We present a constraint-based language which enables us to define queries in a declarative way addressing patterns sets and global patterns. By specifying what the task is, rather than providing how the solution should be computed, it is easy to process by stepwise refinements to successfully discover global patterns. The usefulness of the approach is highlighted by several examples coming from the clustering based on associations. All primitive constraints of the language are modeled and solved using the SAT framework. We illustrate the efficiency of our approach through several experiments.
\end{abstract}

\section{INTRODUCTION}

The process of extracting useful patterns from data, called pattern mining, is an important tool for data analysis and has been used in a wide range of applications and domains. A large amount of work has been developed and many pattern extraction problems are now identified and understood from both theoretical and computational perspectives. Local pattern discovery has become a growing field [19] and several paradigms are available for producing extensive collections of patterns such as the constraint-based pattern mining [20] or condensed representations of patterns [3]. Because of the exhaustive nature of the techniques, the pattern collections provide a fairly complete picture of the information content of the data. However, the approach suffers from limitations. First, the collections of patterns still remain too large for an individual and global analysis performed by the data analyst. Secondly, the so-called local patterns represent fragmented information whereas patterns expected by the data analyst require to consider simultaneously several local patterns. That is why combining local patterns to get global patterns is highly attractive.

The data mining literature includes several methods to take into account the relationships between patterns and produce global patterns or pattern sets [4], [9]. Recent approaches - constraint-based pattern set mining [4], pattern teams [15] and selecting patterns according to the added value of a new pattern given the currently selected patterns [2] - aim at reducing the redundancy by selecting patterns from the initial large set of local patterns on the basis of their usefulness in the context of the other selected patterns. Nevertheless, these methods are mainly based on the reduction of the redundancy or specific aims such as classification processes. The difficulty of the task may explain the use of heuristic functions and the lack of complete and correct methods to mine global patterns. Indeed, mining local patterns requires the exploration of a large search space but mining global patterns is even harder because solutions satisfying each pattern must be compared. Clearly, the lack of generic approaches restrains the discovery of useful global patterns because the user has to develop a new method each time he wants to extract a new kind of global patterns. It explains why this issue deserves our attention.

In this paper, we propose a constraint-based language to discover patterns combining several local patterns. The key idea is to propose a declarative and generic approach to ask queries: the user models a problem by specifying a set of constraints and expresses his queries thanks to constraints over terms built from constants, variables, operators, and function symbols. Queries and built-in constraints of the language are encoded and solved using the SAT framework. Our approach takes benefit of the recent progress on cross-fertilization between data mining and Constraint Programming [11], [12], [13], [22]. The definition of a constraint-based language offers the great advantage to provide a declarative method to address different pattern mining problems: it is enough to change the specification in term of constraints. We illustrate the approach by several examples coming from the clustering based on associations. With simple query refinements, the data analyst is able to easily produce clusterings satisfying different properties. By specifying what the task is, rather than providing how the solution should be computed, the process greatly facilitates the search of global patterns and the discovery of knowledge.

This paper is organized as follows. Section II describes the constraint-based language and shows how queries and constraints can be defined using terms and built-in constraints. Starting from the clustering example, Section III depicts the process of successive refinements which enables us to easily address several kinds of clustering and the discovery of global models. Section IV describes how queries and built-in 
constraints of the language are modeled and solved using the SAT framework. Section V demonstrates the efficiency of our approach through several experiments. We review related work in Section VI.

\section{A Constraint-BASED LAnguAGE}

This section describes the constraint-based language we propose. Terms are built using constants, variables, operators, and function symbols. Constraints are relations over terms that can be satisfied or not. The data analyst can define new function symbols. We show how queries and constraints can be written using built-in constraints.

\section{A. Definitions and example}

Let $\mathcal{I}$ be a set of $n$ distinct literals called items, an itemset (or pattern) is a non-null subset of $\mathcal{I}$. The language of itemsets corresponds to $\mathcal{L}_{\mathcal{I}}=2^{\mathcal{I}} \backslash \emptyset$. A transactional dataset is a multi-set of $m$ itemsets of $\mathcal{L}_{\mathcal{I}}$. Each itemset, usually called a transaction or object, is a database entry. For instance, Table I gives a transactional dataset $\mathcal{T}$ with $m=11$ transactions $t_{1}, \ldots, t_{11}$ described by $n=8$ items $A, B, C, D, E, F, G, H$. Interestingness measures such as the frequency and the area [8] are commonly used to evaluate the relevance of patterns.

Definition 1. (frequency) The frequency of a pattern $X_{i}$ is the number of transactions that $X_{i}$ covers in $\mathcal{T}: \operatorname{freq}\left(X_{i}\right)$ $=\left|\left\{t \in \mathcal{T} \mid X_{i} \subseteq t\right\}\right|$.

Definition 2. (area) Let $X_{i}$ be a pattern, area $\left(X_{i}\right)=$ freq $\left(X_{i}\right) \times \operatorname{size}\left(X_{i}\right)$ where $\operatorname{size}\left(X_{i}\right)$ denotes the cardinality of $X_{i}$.

From our running example (cf. Table I), freq $(\{A, E\})=$ 3 and $\operatorname{freq}(\{C, F, G, H\})=1$. The frequency constraint freq $\left(X_{i}\right) \geq \operatorname{minfr}$ focuses on patterns occurring in the dataset a number of times exceeding a given minimal threshold minfr. On the other hand, 9 patterns satisfy the constraint area $\left(X_{i}\right) \geq 6:\{A, E, G\},\{B, E, G\},\{C, E, G\}$, $\{C, E, H\},\{E, G\},\{C, E\},\{C, H\},\{E\},\{G\}$. The goal of constraint-based pattern mining is to discover all the patterns of $\mathcal{L}_{\mathcal{I}}$ satisfying a given constraint. The rest of this section depicts our proposition to express constraints. Contrary to the previous examples, several patterns can be involved in the following constraints.

\section{B. Terms}

Terms are built from constants, variables, operators, and function symbols.

- constants are either numerical values (as threshold minfr), or items (as $A$ ) or patterns (as $\{A, B\}$ ) or transactions (as $t_{7}$ ).

- variables, noted $X_{i}$, for $1 \leq i \leq k$, represent the unknown patterns.

- operators:

- set operators as $\cap, \cup, \backslash, \ldots$

- numerical operators as,,$+- \times, /, \ldots$

\begin{tabular}{|c|c|c|c|c|c|c|c|}
\hline Trans. & \multicolumn{7}{|c|}{ Items } \\
\hline$t_{1}$ & $\bar{A}$ & & & & $\mathrm{~F}$ & & \\
\hline$t_{2}$ & A & & & E & F & & \\
\hline$t_{3}$ & A & & & $\mathrm{E}$ & & G & \\
\hline$t_{4}$ & A & & & $\mathrm{E}$ & & G & \\
\hline$t_{5}$ & & B & & E & & G & \\
\hline$t_{6}$ & & B & & $\mathrm{E}$ & & G & \\
\hline$t_{7}$ & & & C & E & & G & \\
\hline$t_{8}$ & & & $\mathrm{C}$ & $\mathrm{E}$ & & $\mathrm{G}$ & \\
\hline$t_{9}$ & & & $\mathrm{C}$ & $\mathrm{E}$ & & & $\mathrm{H}$ \\
\hline$t_{10}$ & & & $\mathrm{C}$ & $\mathrm{E}$ & & & $\mathrm{H}$ \\
\hline$t_{11}$ & & & C & & $\mathrm{F}$ & G & $\mathrm{H}$ \\
\hline
\end{tabular}

Table I

TRANSACTIONAL DATASET $\mathcal{T}$.

- function symbols involving one or several patterns: freq/1, size/1, cover/1, overlapItems/2, overlapTransactions/2,..

Examples of terms:

- $\operatorname{freq}\left(X_{1}\right) \times \operatorname{size}\left(X_{1}\right)$ (i.e. the area of $\left.X_{1}\right)$

- $\operatorname{freq}\left(X_{1} \cup X_{2}\right) \times \operatorname{size}\left(X_{1} \cap X_{2}\right)$ (i.e., the overlapping between area $\left(X_{1}\right)$ and area $\left(X_{2}\right)$ )

- $\operatorname{freq}\left(X_{1}\right)$ - freq $\left(X_{2}\right)$

1) Built-in function symbols: The constraint based language owns predefined (built-in) function symbols ${ }^{1}$ like:

- $\operatorname{cover}\left(X_{i}\right)=\left\{t \mid t \in \mathcal{T}, X_{i} \subseteq t\right\}$ is the set of transactions covered by $X_{i}$.

- $\operatorname{freq}\left(X_{i}\right)=\left|\left\{t \mid t \in \mathcal{T}, X_{i} \subseteq t\right\}\right|$

- $\operatorname{size}\left(X_{i}\right)=\left|\left\{j \mid j \in \mathcal{I}, j \in X_{i}\right\}\right|$

- overlapItems $\left(X_{i}, X_{j}\right)=\left|X_{i} \cap X_{j}\right|$ is the number of items shared by both $X_{i}$ and $X_{j}$.

- overlapTransactions $\left(X_{i}, X_{j}\right)=\mid \operatorname{cover}\left(X_{i}\right) \cap$ cover $\left(X_{j}\right) \mid$ is the number of transactions covered by both $X_{i}$ and $X_{j}$.

2) User-defined function symbols: The data analyst can define new function symbols using constants, variables, operators and existing function symbols (built-in or previously defined ones). Examples:

- $\operatorname{area}\left(X_{i}\right)=\operatorname{freq}\left(X_{i}\right) \times \operatorname{size}\left(X_{i}\right)$

- coverage $\left(X_{i}, X_{j}\right)=$ freq $\left(X_{i} \cup X_{j}\right) \times \operatorname{size}\left(X_{i} \cap X_{j}\right)$

- interestingness measures can be straightforwardly defined. Here is the example of the growth-rate wellused in contrast mining [21]. Let $D_{1}, D_{2} \subset \mathcal{T}$ be 2 sets of transactions (i.e., classes) and freq' $\left(X_{i}, D_{j}\right)$ the frequency of $X_{i}$ into $D_{j}$, the growth-rate of $X_{i}$ in $D_{1}$ is $\operatorname{gr}_{1}\left(X_{i}\right)=\frac{\left|D_{2}\right| \times \text { freq }^{\prime}\left(X_{i}, D_{1}\right)}{\left|D_{1}\right| \times \text { freq }^{\prime}\left(X_{i}, D_{2}\right)}$

\section{Constraints and Queries}

Constraints are relations over terms that can be satisfied or not. There are three kinds of built-in constraints:

${ }^{1}$ Only function symbols used in Section III are introduced in this paper. 
- numerical constraints like: $<, \leq,=, \neq, \geq,>, \ldots$ Examples:

- $\operatorname{freq}\left(X_{1}\right) \leq 10$

- $\operatorname{size}\left(X_{2}\right)=2 \times \operatorname{size}\left(X_{3}\right)$

- $\operatorname{area}\left(X_{1}\right)<\operatorname{size}\left(X_{2}\right) \times \operatorname{freq}\left(X_{3}\right)$

- set constraints like: $=, \neq, \in, \notin, \subset, \subseteq, \ldots$

Examples:

- $A \in X_{1}$

- $X_{1} \cup X_{2} \subset X_{3}$

- $X_{1}=X_{2} \cap X_{4}$

- dedicated constraints like:

- closed $\left(X_{i}\right)$ is satisfied iff $X_{i}$ is a closed ${ }^{2}$ pattern.

- coverTransactions $\left(\left[X_{1}, \ldots, X_{k}\right]\right)$ is satisfied iff each transaction is covered by at least one pattern (i.e. $\left.\bigcup_{1 \leq i \leq k} \operatorname{cover}\left(X_{i}\right)=\mathcal{T}\right)$,

- coverItems $\left(\left[X_{1}, \ldots, X_{k}\right]\right)$ is satisfied iff every item belongs to at least one pattern (i.e. $\bigcup_{1 \leq i \leq k} X_{i}=\mathcal{I}$ ).

- canonical $\left(\left[X_{1}, \ldots, X_{k}\right]\right)$ is satisfied iff for all $i$ s.t. $1 \leq i<k$, pattern $X_{i}$ is less than pattern $X_{i+1}$ with respect to the lexicographic order.

Queries are formulae built using constraints and logical connectors: $\wedge$ (conjunction) and $\vee$ (disjunction).

\section{MINING BY REFINING CONTRAINT-BASED QUERIES}

A major strength of our approach is to provide a simple and efficient way to declare and refine queries. In practice, the data analyst starts by writing a first query $Q_{1}$. Then, he successively refines the query (deriving $Q_{i+1}$ from $Q_{i}$ ) until he considers that relevant information has been extracted. We illustrate this approach with the clustering problem. Clustering aims at partitioning data into groups (clusters) so that transactions are similar inside each cluster but different between clusters [7]. We selected clustering because it is an important and popular data mining task and, by nature, clustering proceeds by iteratively refining queries until a satisfactory solution is found. Our approach is also wellsuited to integrate constraints handled in constraint-based clustering [1].

\section{A. Modeling a clustering query}

The closed patterns are well-designed for clustering based on associations because a closed pattern gathers the maximum amount of similarity between a set of transactions. Thus, a closed pattern is a candidate cluster. The standard clustering problem can then be formalized as: "to find a set of $k$ closed patterns $X_{1}, X_{2}, \ldots, X_{k}$ (i.e., clusters) covering all transactions without any overlap on these transactions".

Our constraint-based language offers the constraints to express this query: the $\operatorname{closed}\left(X_{i}\right)$ constraints enforce each unknown pattern $X_{i}$ to be closed, the coverTransactions $\left(\left[X_{1}, \ldots, X_{k}\right]\right)$ constraint ensures to

${ }^{2}$ Let $T r_{i}$ be the set of transactions covered by pattern $X_{i} . X_{i}$ is closed iff $X_{i}$ is the largest $(\subset)$ pattern covering $T r_{i}$.

\begin{tabular}{c|l|l|l} 
Sol. & $X_{1}$ & $X_{2}$ & $X_{3}$ \\
\hline$s_{1}$ & $\{\mathrm{C}, \mathrm{F}, \mathrm{G}, \mathrm{H}\}$ & $\{\mathrm{E}\}$ & $\{\mathrm{A}, \mathrm{D}, \mathrm{F}\}$ \\
$s_{2}$ & $\{\mathrm{~A}, \mathrm{~F}\}$ & $\{\mathrm{C}, \mathrm{H}\}$ & $\{\mathrm{E}, \mathrm{G}\}$ \\
$s_{3}$ & $\{\mathrm{C}, \mathrm{E}, \mathrm{H}\}$ & $\{\mathrm{E}, \mathrm{G}\}$ & $\{\mathrm{F}\}$ \\
$s_{4}$ & $\{\mathrm{~A}, \mathrm{~F}\}$ & $\{\mathrm{C}, \mathrm{E}, \mathrm{H}\}$ & $\{\mathrm{G}\}$ \\
$s_{5}$ & $\{\mathrm{~A}\}$ & $\{\mathrm{B}, \mathrm{E}, \mathrm{G}\}$ & $\{\mathrm{C}\}$ \\
\hline
\end{tabular}

Table II

SET OF DIFFERENT CLUSTERINGS.

\begin{tabular}{c|l|l|l} 
Sol. & $X_{1}$ & $X_{2}$ & $X_{3}$ \\
\hline$s_{1}^{\prime}$ & $\{\mathrm{C}, \mathrm{F}, \mathrm{G}, \mathrm{H}\}$ & $\{\mathrm{E}\}$ & $\{\mathrm{F}\}$ \\
$s_{2}^{\prime}$ & $\{\mathrm{A}, \mathrm{D}, \mathrm{F}\}$ & $\{\mathrm{C}, \mathrm{F}, \mathrm{G}, \mathrm{H}\}$ & $\{\mathrm{E}\}$ \\
$s_{3}^{\prime}$ & $\{\mathrm{A}, \mathrm{F}\}$ & $\{\mathrm{C}, \mathrm{F}, \mathrm{G}, \mathrm{H}\}$ & $\{\mathrm{E}\}$ \\
$s_{4}^{\prime}$ & $\{\mathrm{A}, \mathrm{E}, \mathrm{F}\}$ & $\{\mathrm{E}\}$ & $\{\mathrm{F}\}$ \\
$s_{5}^{\prime}$ & $\{\mathrm{A}, \mathrm{D}, \mathrm{F}\}$ & $\{\mathrm{E}\}$ & $\{\mathrm{F}\}$ \\
$s_{6}^{\prime}$ & $\{\mathrm{A}\}$ & $\{\mathrm{B}, \mathrm{E}, \mathrm{G}\}$ & $\{\mathrm{C}\}$ \\
$s_{7}^{\prime}$ & $\{\mathrm{A}, \mathrm{F}\}$ & $\{\mathrm{C}, \mathrm{E}, \mathrm{H}\}$ & $\{\mathrm{G}\}$ \\
$s_{8}^{\prime}$ & $\{\mathrm{A}, \mathrm{F}\}$ & $\{\mathrm{C}, \mathrm{H}\}$ & $\{\mathrm{G}\}$ \\
$s_{9}^{\prime}$ & $\{\mathrm{A}, \mathrm{F}\}$ & $\{\mathrm{C}, \mathrm{H}\}$ & $\{\mathrm{E}, \mathrm{G}\}$ \\
$s_{10}^{\prime}$ & $\{\mathrm{C}, \mathrm{E}, \mathrm{H}\}$ & $\{\mathrm{E}, \mathrm{G}\}$ & $\{\mathrm{F}\}$ \\
$s_{11}^{\prime}$ & $\{\mathrm{C}, \mathrm{H}\}$ & $\{\mathrm{E}, \mathrm{G}\}$ & $\{\mathrm{F}\}$ \\
$s_{12}^{\prime}$ & $\{\mathrm{C}, \mathrm{H}\}$ & $\{\mathrm{F}\}$ & $\{\mathrm{G}\}$ \\
$s_{13}^{\prime}$ & $\{\mathrm{C}, \mathrm{E}, \mathrm{H}\}$ & $\{\mathrm{F}\}$ & $\{\mathrm{G}\}$ \\
\hline
\end{tabular}

Table III

SET OF DIFFERENT CLUSTERINGS FOR QUERY $Q_{4}$.

cover all the transactions. To avoid an overlap between transactions, we add for each couple of patterns $\left(X_{i}, X_{j}\right)$ s.t. $i<j$ the overlapTransactions $\left(X_{i}, X_{j}\right)=0$ constraint. This constraint states that there exists no transaction covered by both $X_{i}$ and $X_{j}$.

Moreover, a clustering problem intrinsically owns a lot of symmetrical solutions. Let $s=\left(p_{1}, p_{2}, \ldots, p_{k}\right)$ be a solution containing $k$ patterns $p_{i}$, any permutation $\sigma$ of these $k$ patterns $\sigma(s)=\left(p_{\sigma(1)}, p_{\sigma(2)}, \ldots, p_{\sigma(k)}\right)$ is also a solution. The canonical $\left(\left[X_{1}, \ldots, X_{k}\right]\right)$ constraint is used to avoid computing symmetrical solutions. This constraint ensures that, for all $i$ s.t. $1 \leq i<k$, pattern $X_{i}$ is before pattern $X_{i+1}$ with respect to the lexicographic order. The canonical $\left(\left[X_{1}, \ldots, X_{k}\right]\right)$ constraint plays an important role. As for a clustering involving $k$ clusters, the number of symmetrical solutions is $k$ !, it is crucial to break the symmetries to avoid obtaining a huge number of redundant solutions. Moreover, this constraint performs an efficient filtering by drastically reducing the size of the search space.

Finally, we get the following query $\left(Q_{1}\right)$ modeling the initial clustering problem:

$$
\left\{\begin{array}{l}
\wedge_{1 \leq i \leq k} \text { Closed }\left(X_{i}\right) \wedge \\
\text { coverTransactions }\left(\left[X_{1}, \ldots, X_{k}\right]\right) \wedge \\
\wedge_{1 \leq i<j \leq k} \text { overlapTransactions }\left(X_{i}, X_{j}\right)=0 \wedge \\
\text { canonical }\left(\left[X_{1}, \ldots, X_{k}\right]\right)
\end{array}\right.
$$

On our running example, with $k=3$ patterns, query $Q_{1}$ provides 5 solutions (see Table II). 


\section{B. Refining queries}

By only refining queries on a clustering, the data analyst can easily produce other clusterings satisfying different properties. This section illustrates this feature of our approach that facilitates the building of global patterns and the discovery of knowledge. From the initial query $Q_{1}$, we derive queries $Q_{2}$ and $Q_{3}$ avoiding clusterings with nonfrequent patterns and clusterings with small size patterns.

1) Removing solutions with non-frequent patterns: When a cluster has a low frequency, it lacks of representativity and the clustering is not considered as reliable. From $Q_{1}$, it is easy to add frequency constraints ensuring that each pattern is frequent. With a frequency threshold $\delta_{1}=2$, we get $Q_{2}$ :

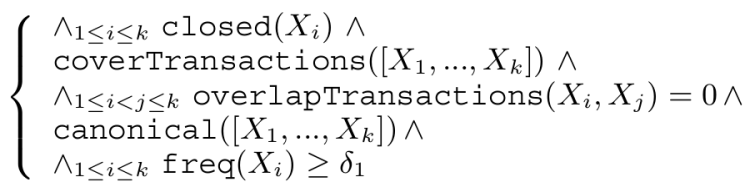

Pattern $\{C, F, G, H\}$ of solution $s_{1}$ has a frequency of 1 and thus is removed. With $Q_{2}$, there remain 4 solutions $\left(s_{2}\right.$, $s_{3}, s_{4}$, and $s_{5}$, see Table II).

2) Removing solutions with small size patterns: A clustering with at least one cluster $X_{i}$ of small size ${ }^{3}$ is not considered as useful because $X_{i}$ does not ensure enough similarity between transactions associated to $X_{i}$. It is simple to add constraints requiring that the size of each pattern is higher than a minimal size. From $Q_{2}$, with a minimal size threshold $\delta_{2}=2$, we obtain the query $Q_{3}$ :

$$
\left\{\begin{array}{l}
\wedge_{1 \leq i \leq k} \operatorname{closed}\left(X_{i}\right) \wedge \\
\text { coverTransactions }\left(\left[X_{1}, \ldots, X_{k}\right]\right) \wedge \\
\wedge_{1 \leq i<j \leq k} \text { overlapTransactions }\left(X_{i}, X_{j}\right)=0 \wedge \\
\text { canonical }\left(\left[X_{1}, \ldots, X_{k}\right]\right) \wedge \\
\wedge_{1 \leq i \leq k} \text { freq }\left(X_{i}\right) \geq \delta_{1} \wedge \\
\wedge_{1 \leq i \leq k} \operatorname{size}\left(X_{i}\right) \geq \delta_{2}
\end{array}\right.
$$

With the query $Q_{3}$, there is only one solution: $s 2$ with $X_{1}=\{A, F\}, X_{2}=\{C, H\}$ and $X_{3}=\{E, G\}$ (cf. Table II).

\section{Solving other Clustering Problems}

In the same way, it is easy to express other clustering problems [1] such as soft clustering, co-clustering, and soft co-clustering.

1) Soft clustering: This problem is a relaxed version of the clustering where small overlaps on transactions (less than a threshold $\delta_{T}$ ) are allowed. The query $Q_{4}$ (soft version of $Q_{1}$ ) models this problem:

$$
\left\{\begin{array}{l}
\wedge_{1 \leq i \leq k} \text { closed }\left(X_{i}\right) \wedge \\
\text { coverTransactions }\left(\left[X_{1}, \ldots, X_{k}\right]\right) \wedge \\
\wedge_{1 \leq i<j \leq k} \text { overlapTransactions }\left(X_{i}, X_{j}\right) \leq \delta_{T} \wedge \\
\text { canonical }\left(\left[X_{1}, \ldots, X_{k}\right]\right)
\end{array}\right.
$$

\footnotetext{
${ }^{3}$ Moreover, clustering with clusters of size 1 often reflects values coming from the binarization of an attribute (such as $A, B$ and $C$ in Table I) and are useless.
}

With $k=3$ and a maximal overlap between transactions $\delta_{T}=1, Q_{4}$ produces 13 solutions (see Table III).

With $s_{1}^{\prime}$, the overlaps are the transaction $t_{11}$ (covered by $X_{1}$ and $X_{3}$ ) and the transaction $t_{2}$ (covered by $X_{2}$ and $X_{3}$ ) (see Tables III and I). Removing solutions with non frequent patterns (with $\delta_{1}=2$ ) leads to 8 solutions (from $s_{6}^{\prime}$ to $s_{13}^{\prime}$ ). By adding a minimal size constraint $\delta_{2}=2$, only the solution $s_{9}^{\prime}$ remains (which is also the solution $s_{2}$ of the initial clustering problem, see Section III-B2).

2) Co-clustering: The co-clustering task consists in finding $k$ clusters covering both the set of transactions and the set of items, without any overlap on transactions or on items. Query $Q_{5}$ expresses this problem:

$$
\left\{\begin{array}{l}
\wedge_{1 \leq i \leq k} \text { Closed }\left(X_{i}\right) \wedge \\
\text { coverTransactions }\left(\left[X_{1}, \ldots, X_{k}\right]\right) \wedge \\
\wedge_{1 \leq i<j \leq k} \text { overlapTransactions }\left(X_{i}, X_{j}\right)=0 \wedge \\
\text { coverItems }\left(\left[X_{1}, \ldots, X_{k}\right]\right) \wedge \\
\wedge_{1 \leq i<j \leq k} \text { overlapItems }\left(X_{i}, X_{j}\right)=0 \wedge \\
\text { canonical }\left(\left[X_{1}, \ldots, X_{k}\right]\right)
\end{array}\right.
$$

3) Soft co-clustering: This problem is a relaxed version of the co-clustering, allowing small overlaps on transactions (less than $\delta_{T}$ ) and on items (less than $\delta_{I}$ ). The query $Q_{6}$ (soft version of $Q_{4}$ and $Q_{5}$ ) models this task:

$$
\left\{\begin{array}{l}
\wedge_{1 \leq i \leq k} \text { closed }\left(X_{i}\right) \wedge \\
\text { coverTransactions }\left(\left[X_{1}, \ldots, X_{k}\right]\right) \wedge \\
\wedge_{1 \leq i<j \leq k} \text { overlapTransactions }\left(X_{i}, X_{j}\right) \leq \delta_{T} \wedge \\
\text { coverItems }\left(\left[X_{1}, \ldots, X_{k}\right]\right) \wedge \\
\wedge_{1 \leq i<j \leq k} \text { overlapItems }\left(X_{i}, X_{j}\right) \leq \delta_{I} \wedge \\
\text { canonical }\left(\left[X_{1}, \ldots, X_{k}\right]\right)
\end{array}\right.
$$

4) Balanced clustering: In clustering, we generally prefer solutions in which the frequencies of the clusters do not differ too much from each other. Query $Q_{7}$ describes clusterings with balanced frequencies. For any couple of clusters $\left(X_{i}, X_{j}\right)$, their difference of frequencies must be lower than a threshold $\Delta \times m$ where $\Delta$ is a percentage. Looking for clusterings with balanced size clusters could be achieved in a same way.

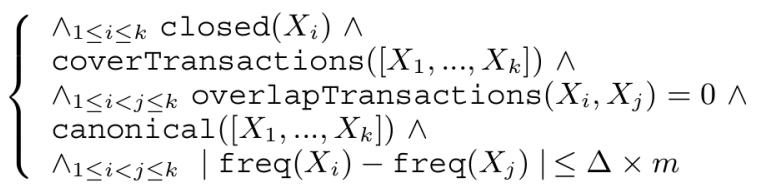

\section{Modeling and Solving as a SAT Problem}

Satisfiability (SAT) is the problem of determining if the variables of a given boolean formula can be assigned in such a way as to make the formula be evaluated to True. A formula is in conjunctive normal form (CNF) if it is a conjunction of clauses, where a clause is a disjunction of literals and a literal is either a variable $x_{i}$ or its negation $\neg x_{i}$.

Even if the SAT problem is NP-complete, efficient and scalable algorithms for SAT, that were developed over the 
last decade, have contributed to dramatic advances in the ability to automatically solve problem instances involving tens of thousands of variables and millions of constraints. That is why, we have chosen to transform a query into a $\mathrm{CNF}$ and then use a SAT solver to find its solutions.

In the remainder of this section, let $\left(d_{t, i}\right)$ be the $(m, n)$ boolean matrix where $\left(d_{t, i}=\right.$ True $)$ iff $(i \in t)$. Queries are modeled in two steps. First, unknown patterns are modeled using boolean variables and matrix $\left(d_{t, i}\right)$. Then, each built-in constraint its expressed using a CNF.

\section{A. Modeling unknown patterns}

Let $X_{1}, X_{2}, \ldots, X_{k}$ be the $k$ patterns we are looking for. In a same way as [13], [22], the link between the data set $\mathcal{T}$ and an unknown pattern $X_{j}$ is performed by introducing two kinds of boolean variables:

- $X_{1, j}, X_{2, j}, \ldots, X_{n, j}$ s.t. $\left(X_{i, j}=\right.$ True $)$ iff $\left(i \in X_{j}\right)$

- $T_{1, j}, T_{2, j}, \ldots, T_{m, j}$ s.t. $\left(T_{t, j}=\right.$ True $)$ iff $\left(X_{j} \subset t\right)$

The relation $\left(X_{j} \subset t\right)$ can be transformed into the following CNF:

$$
\bigwedge_{\left\{\in \mathcal{I} \mid \neg d_{t, i}\right\}} \neg X_{i, j}
$$

The relationship between $X_{j}$ and $\mathcal{T}$ is modeled by stating that, for each transaction $t,\left(T_{t, j}=\right.$ True $)$ iff $X_{j}$ covers $t$ :

$$
\forall t \in \mathcal{T}, T_{t, j} \Leftrightarrow\left(X_{j} \subset t\right)
$$

Using Eq. 1, the left to right implication of Eq. 2 can be transformed into the following $\mathrm{CNF}$ :

$$
\bigwedge_{t \in \mathcal{T}}\left(\bigwedge_{\left\{i \in \mathcal{I} \mid \neg d_{t, i}\right\}}\left(\neg T_{t, j} \vee \neg X_{i, j}\right)\right)
$$

Using Eq. 1, the right to left implication of Eq. 2 can be transformed into the following $\mathrm{CNF}$ :

$$
\bigwedge_{t \in \mathcal{T}}\left(\bigvee_{\left\{i \in \mathcal{I} \mid d_{t, i}\right\}} X_{i, j} \vee T_{t, j}\right)
$$

Finally, Eq. 3 and Eq. 4 must hold for every $X_{j}, 1 \leq j \leq k$. So, the SAT encoding of a query with $k$ unknown patterns requires $k \times m \times n$ binary clauses.

\section{B. Constraints as boolean formulae}

This section provides the boolean formulae associated to built-in constraints. The following ones have a straightforward encoding:

- $X_{p}=X_{q} \rightarrow \wedge_{i \in \mathcal{I}}\left(X_{i, p} \Leftrightarrow X_{i, q}\right)$

- $i_{o} \in X_{p} \rightarrow X_{i_{0}, p}$

- $X_{p} \cap X_{q}=X_{r} \rightarrow \wedge_{i \in \mathcal{I}}\left(X_{i, r} \Leftrightarrow X_{i, p} \wedge X_{i, q}\right)$

- $X_{p} \cup X_{q}=X_{r} \rightarrow \wedge_{i \in \mathcal{I}}\left(X_{i, r} \Leftrightarrow X_{i, p} \vee X_{i, q}\right)$

- $X_{p} \backslash X_{q}=X_{r} \rightarrow \wedge_{i \in \mathcal{I}}\left(X_{i, r} \Leftrightarrow X_{i, p} \wedge \neg X_{i, q}\right)$

- coverItems $\left(\left[X_{1}, \ldots, X_{k}\right]\right) \rightarrow \wedge_{i \in \mathcal{I}}\left(\vee_{j \in[1 . . k]} X_{i, j}\right)$

- coverTransactions $\left(\left[X_{1}, \ldots, X_{k}\right]\right) \rightarrow \wedge_{t}\left(\vee_{j} T_{t, j}\right)$

All threshold constraints are modelled using the sorting network approach (for technical details, see [6]) in order

\begin{tabular}{c|ccc} 
dataset & \#transactions & \#items & density \\
\hline Australian & 690 & 125 & 0.40 \\
Mushroom & 8124 & 119 & 0.19 \\
Soybean & 630 & 50 & 0.32 \\
Primary-Tumor & 336 & 36 & 0.48 \\
Zoo & 101 & 36 & 0.44 \\
\hline Meningitis & 329 & 82 & 0.26 \\
\multicolumn{2}{c}{ Description OF THE DATASETS. }
\end{tabular}

to prevent prohibitive grounding. Using such an encoding, the size of the CNF modelling a threshold constraint is independent from the value of the threshold but depends on the maximal value for the considered measure. For example, the size of the $\mathrm{CNF}$ for constraint $\left(\operatorname{freq}\left(X_{i}\right) \geq \delta_{1}\right)$ is $O(m \times \log (m))$, and does not depend on $\delta_{1}$. The size of the CNF for constraint $\left(\operatorname{size}\left(X_{j}\right) \leq \delta_{2}\right)$ is $O(n \times \log (n))$, and does not depend on $\delta_{2}$. Threshold constraints for measures like overlapItems and overlapTransactions are encoded in the same way.

\section{Ensuring completeness while using a SAT solver}

Given a CNF, SAT solvers either find one instanciation (and only one) for the variables evaluating the formula to True, or prove there is no such an instanciation. In order to ensure the completeness of our approach, restarts are performed.

Let $\mathcal{F}$ be the CNF modeling a query $Q . \mathcal{F}$ is the conjunction of the CNF associated to the modeling of unknown patterns (see Section IV-A) and the CNFs associated to the modeling of the constraints involved in $Q$ (see Section IV-B). Resolution begins with $\mathcal{F}$. Then, after having obtained the $i$-th solution $s_{i}$, its negation $\neg s_{i}$ is added to the (current) $\mathrm{CNF}$ and resolution is restarted in order to look for another solution. The process ends when a failure occurs, i.e. when all solutions have been found.

Using restarts may seem too naive, but in practice is powerful (see experiments in Section V-A). This is because the $\mathrm{CNF} \mathcal{F}$ contains much binary clauses (see Section IV-A), and so, filtering by unit propagation will be very effective.

The SAT solver Minisat ${ }^{4}$ [5] has been used for experiments, because its implementation proved easy to modify and Minisat is one of the most efficient SAT solvers.

\section{EXPERIMENTS}

Recalling that the key contribution of this paper is to propose a constraint-based language to model and mine global patterns in a declarative way. Therefore, the goal of the experiments is to provide better insights on the use of our approach in order to discover global patterns with the example of clustering based on associations.

\footnotetext{
${ }^{4}$ http: //minisat.se/
} 


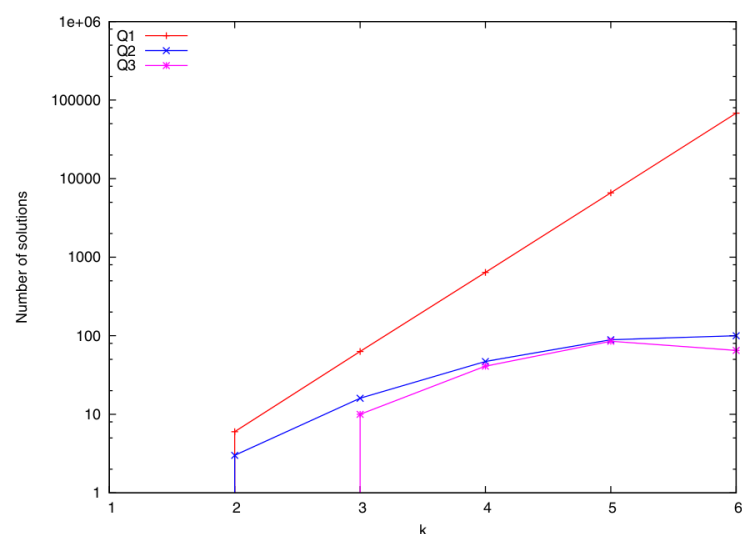

Figure 1. Number of solutions for refinements $Q_{1}$ to $Q_{3}$ (Soybean).

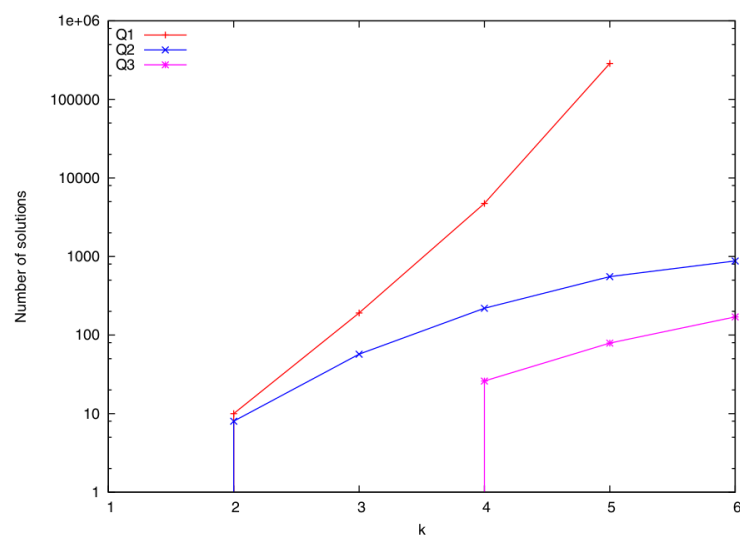

Figure 2. Number of solutions for refinements $Q_{1}$ to $Q_{3}$ (Meningitis).

Experiments were performed on several benchmarks from the UCI repository ${ }^{5}$ and also a real-world dataset Meningitis gathering 329 children hospitalized for acute meningitis. Characteristics of datasets are presented in Table IV. Experiments were conducted on a PC having a 2.83 $\mathrm{GHz}$ Intel Core 2 Duo Processor and 4GB of RAM, running Ubuntu Linux.

\section{A. Clustering Queries}

This section illustrates the successive query refinement depicted in Section III and leading to the query $Q_{3}$.

Fig. 1 (resp. Fig. 2) gives the total number of solutions for queries $Q_{1}, Q_{2}$, and $Q_{3}$ for the dataset Soybean (resp. Meningitis) according to $k$ (i.e., the number of patterns). Applying the stepwise refinements from query $Q_{1}$ to query $Q_{3}$ drastically reduces the number of clusterings and highlights on the most promising ones (note that the $\mathrm{y}$-axis is a logarithmic scale).

Fig. 3 depicts the number of solutions to query $Q_{3}$ on several datasets. In some cases, the query can be satisfied by a large number of solutions and we do not report results when

\footnotetext{
5http://www. ics.uci.edu/ $\sim$ mlearn/MLRepository.html
}

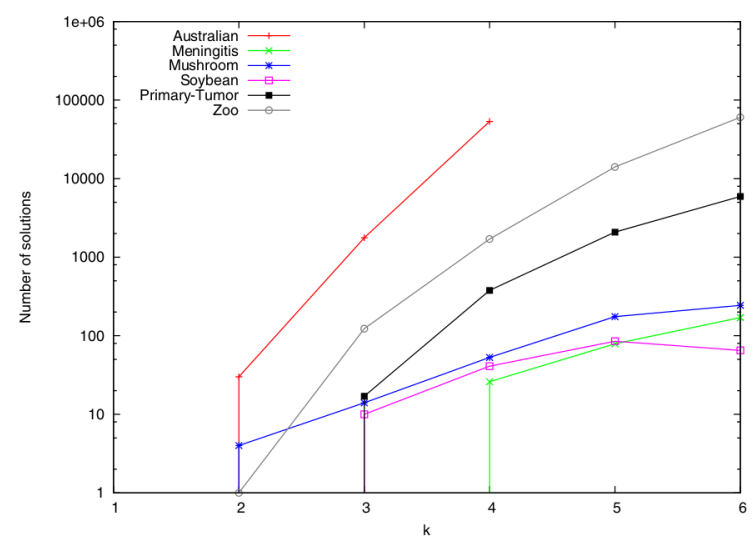

Figure 3. Number of solutions to query $Q_{3}$ on several datasets.

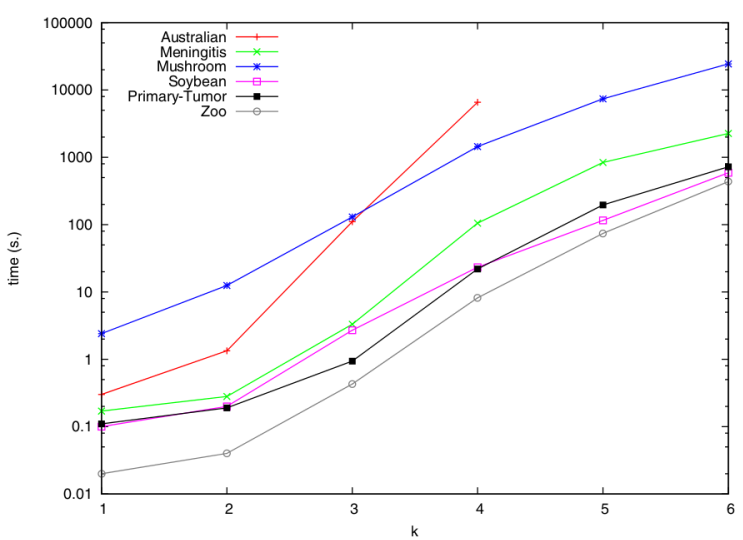

Figure 4. Computing times for query $Q_{3}$ on several datasets.

there are more than one million of solutions. It especially happens with Australian because this dataset provides a huge number of closed patterns. In such situations, the query should be refined (by increasing the minimal frequency and/or size thresholds or adding new constraints).

The completeness of our approach is ensured by restarting the SAT solver. Each time a new solution is found, its negation is stored and added to the current CNF. Memory consumption becomes too high above millions of solutions. From a practical point of view, as the data analyst is interested in extracting a small number of solutions, it does not make sense to perform mining providing millions of patterns. Once again, the stepwise refinement query should be used to avoid these situations and to focus on the most relevant patterns.

Fig. 4 gives the computing times on several datasets, showing the efficiency of our approach. Even for rather large datasets like Mushroom, computing times are still affordable.

\section{B. Soft Clustering}

Fig. 5 and Fig. 6 provide soft clustering results with query $Q_{4}$ on datasets Soybean and Primary-Tumor. 


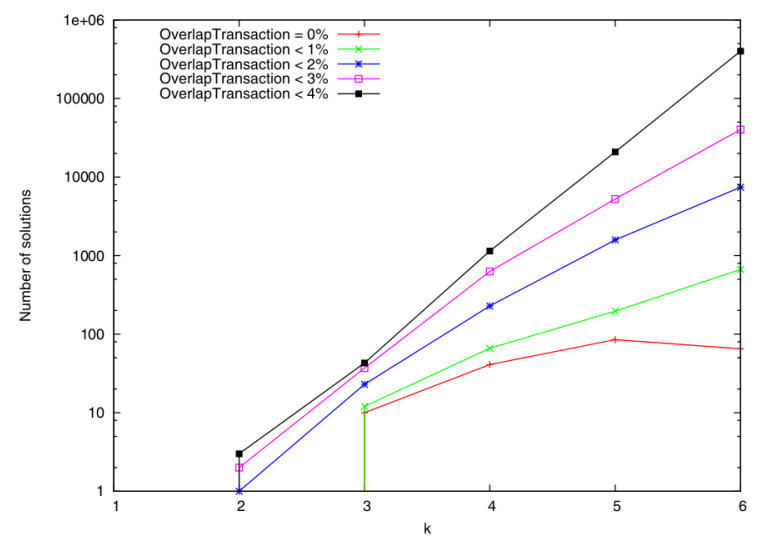

Figure 5. No. of sol. according to $k$ for several $\delta_{T}$ values (Soybean).

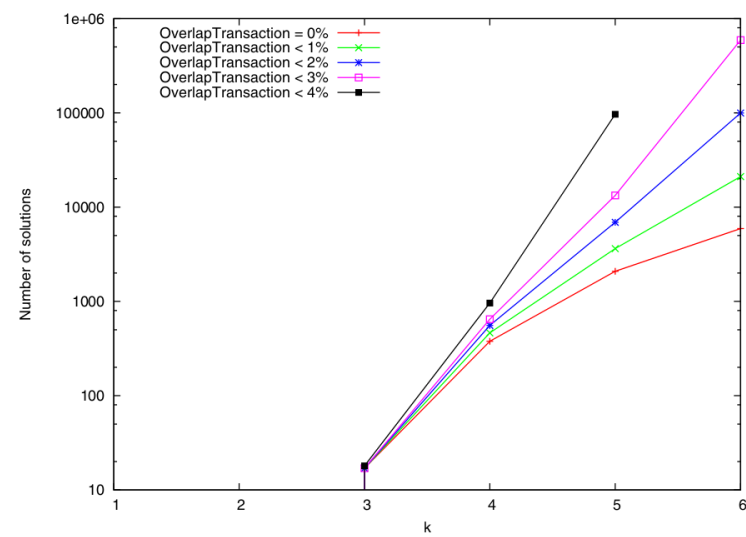

Figure 6. No. of sol. according to $k$ for several $\delta_{T}$ values (Primary-Tumor).

Curves indicate the number of solutions according to $k$ and the maximal overlap threshold $\delta_{T}$ on transactions. As expected, the number of solutions increases with the size of the overlap. The number of solutions can be easily controlled by adjusting (decreasing/increasing) $\delta_{T}$.

\section{Balanced Clustering}

We performed experiments with query $Q_{7}$. Fig. 7 and Fig. 8 display curves indicating the number of solutions on datasets Zoo and Primary-Tumor according to $k$ and $\Delta$. Adding the balanced frequencies constraint strongly reduces the number of solutions. For dataset Zoo, only a gap $\Delta=40 \%$ allows to find a solution for $k=2$. Moreover, with $k=3$ and $k=4$, there is no solution with a gap $\Delta=10 \%$.

\section{RELATED WORK}

The data mining literature includes many approaches to reduce the number of produced patterns such as the condensed representations of patterns [3], the compression of the dataset by exploiting Minimum Description Length Principle [24], the discovery of $k$ representative patterns with probabilistic models for summarizing frequent patterns [18].

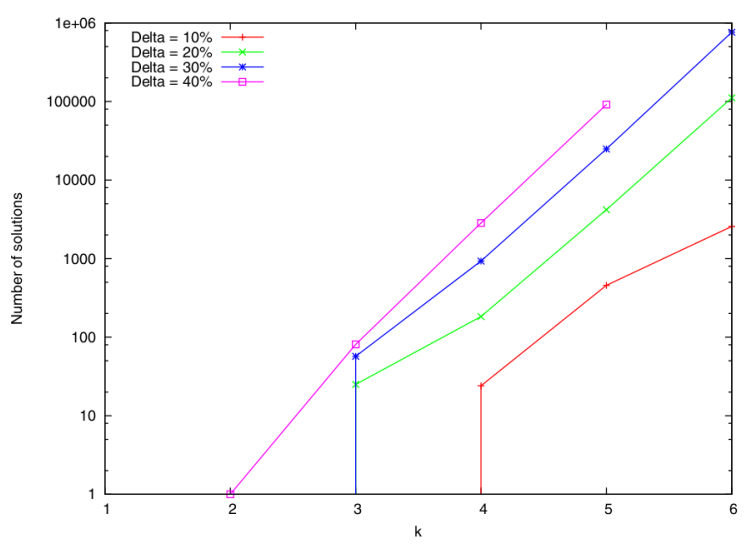

Figure 7. No. of sol. according to $k$ for several $\Delta$ values (Z०O).

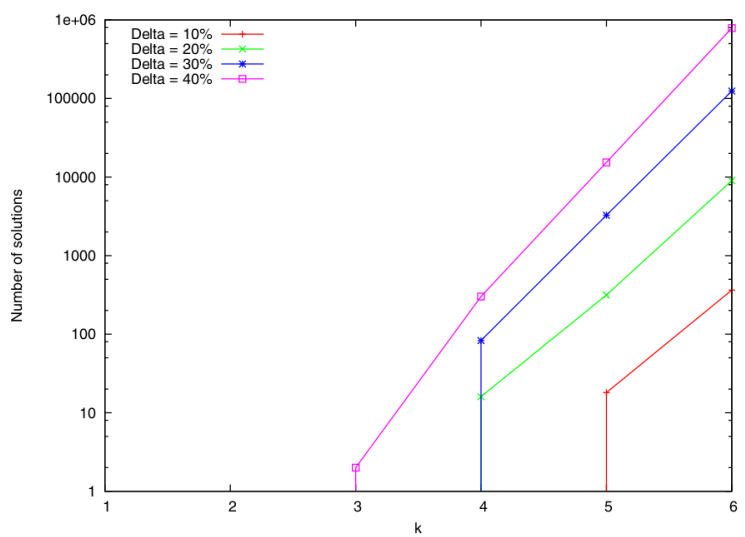

Figure 8. No. of sol. according to $k$ for several $\Delta$ values (Primary-Tumor).

These approaches mainly aim at reducing the redundancy between patterns and often focus on frequent patterns.

Taking into account the relationships between patterns is explicitly required to produce global patterns or pattern sets [2], [4], [9]. A large number of these methods are based on two-step techniques. The first step generates an exhaustive collection of local patterns and then the patterns are heuristically post-processed to select a smaller subset of complementary relevant patterns such as in associative classification [17]. There are few methods without heuristic to mine complete and correct pattern sets or global patterns and in practice running techniques are devoted to specific kinds of global patterns [16], [23]. General data mining frameworks based on the notion of local patterns to design global models are presented in [9], [14]. These frameworks help to analyze and improve current methods in the area.

Recent works [11], [12], [13], [22] have shown the cross-fertilization between data mining and Constraint Programming $(\mathrm{CP})$. Indeed, $\mathrm{CP}$ provides a general declarative methodology for modeling and solving constraint problems. CP facilitates the design of generic methods handling several patterns, that is a key point in pattern set mining. Techniques 
for mining itemsets and n-ary patterns (i.e., the combination of $n$ patterns) have been proposed. Looking for declarative techniques in pattern mining was also recently investigated in [10]: by using relational algebra, the authors propose an algebraic framework for pattern discovery for expressing a wide range of queries.

\section{CONCLUSION AND Future WORK}

We have proposed a constraint-based language allowing to easily express different mining tasks in a declarative way. Thanks to the declarative process, extending or changing the specification to refine the results and discover more relevant patterns or address new global patterns is very simple. Moreover, all constraints can be combined together and new constraints can be added. The efficiency and the flexibility of our approach is shown on several examples coming from clustering based on associations. Thanks to query refinements, the data analyst is able to produce clusterings satisfying different constraints and generating more meaningful clusters.

As future work, we want to enrich our constraint-based language with further constraints to capture and model a wider range of data mining tasks. The scalability of the approach to larger values of $k$ and larger datasets will also be investigated. Another promising direction is to integrate optimisation criteria in our framework.

Acknowledgements: This work is partly supported by the Lower Normandy region and ANR (French Research National Agency) funded projects BINGO2 ANR-07-MDCO014 and FiCOLOFO ANR-10-BLA-0214.

\section{REFERENCES}

[1] P. Berkhin. Survey of clustering data mining techniques. Technical report, Accrue Software, San Jose, CA, USA, 2002.

[2] B. Bringmann and A. Zimmermann. The chosen few: On identifying valuable patterns. In 12th IEEE Int. Conf. on Data Mining (ICDM-07), pages 63-72, 2007.

[3] T. Calders, C. Rigotti, and J-F. Boulicaut. A survey on condensed representations for frequent sets. In ConstraintBased Mining and Inductive Databases, volume 3848 of LNCS, pages 64-80, 2005.

[4] L. De Raedt and A. Zimmermann. Constraint-based pattern set mining. In 7-th SIAM Int. Conf. on Data Mining, 2007.

[5] N. Eén and N. Sörensson. An extensible SAT-solver. In Enrico Giunchiglia and Armando Tacchella, editors, SAT, volume 2919 of LNCS, pages 502-518. Springer, 2003.

[6] Niklas Eén and Niklas Sörensson. Translating pseudo-boolean constraints into sat. JSAT, 2(1-4):1-26, 2006.

[7] D. H. Fisher. Knowledge acquisition via incremental conceptual clustering. Machine Learning, 2(2):139-172, 1987.
[8] F. Geerts, B. Goethals, and T. Mielikäinen. Tiling databases. In Discovery Science, volume 3245 of LNCS, pages 278-289. Springer, 2004.

[9] A. Giacometti, E. Khanjari Miyaneh, P. Marcel, and A. Soulet. A framework for pattern-based global models. In IDEAL'09, volume 5788 of LNCS, pages 433-440, 2009.

[10] A. Giacometti, P. Marcel, and A. Soulet. A relational view of pattern discovery. In DASFAA 2011, volume 6587 of Lecture Notes in Computer Science, pages 153-167, 2011.

[11] T. Guns, S. Nijssen, and L. De Raedt. Evaluating pattern set mining strategies in a constraint programming framework. In PAKDD'11, volume 6635 of LNCS, pages 382-394, 2011.

[12] M. Khiari, P. Boizumault, and B. Crémilleux. Local constraint-based mining and set constraint programming for pattern discovery. In From Local Patterns to Global Models (LeGo-09), ECMLPKDD-09 Workshop, pages 61-76, 2009.

[13] M. Khiari, P. Boizumault, and B. Crémilleux. Constraint programming for mining $\mathrm{n}$-ary patterns. In $C P^{\prime} 10$, volume 6308 of $L N C S$, pages 552-567. Springer-Verlag, 2010.

[14] A. Knobbe, B. Crémilleux, J. Fürnkranz, and M. Scholz. From local patterns to global models: The lego approach to data mining. In Int. Workshop "From Local Patterns to Global Models", ECML/PKDD'08, pages 1-16, 2008.

[15] A. Knobbe and E. Ho. Pattern teams. In PKDD, volume 4213 of LNAI, pages 577-584, 2006.

[16] L. V. S. Lakshmanan, R. T. Ng, J. Han, and A. Pang. Optimization of constrained frequent set queries with 2variable constraints. In SIGMOD'99, pages 157-168, 1999.

[17] B. Liu, W. Hsu, and Y. Ma. Integrating classification and association rules mining. In KDD'98, pages $80-86$, New York, August 1998. AAAI Press.

[18] T. Mielikäinen and H. Mannila. The pattern ordering problem. In PKDD'03, pages 327-338, 2003.

[19] K. Morik, J.-F. Boulicaut, and A. Siebes (eds.), editors. Local Pattern Detection, volume 3539 of LNAI. Springer, 2005.

[20] R. T. Ng, V. S. Lakshmanan, J. Han, and A. Pang. Exploratory mining and pruning optimizations of constrained associations rules. In ACM SIGMOD'98, pages 13-24. ACM Press, 1998.

[21] P. Kralj Novak, N. Lavrac, and G. I. Webb. Supervised descriptive rule discovery: A unifying survey of contrast set, emerging pattern and subgroup mining. Journal of Machine Learning Research, 10:377-403, 2009.

[22] L. De Raedt, T. Guns, and S. Nijssen. Constraint programming for itemset mining. In KDD'08, pages 204-212, 2008.

[23] E. Suzuki. Undirected discovery of interesting exception rules. Int. J. of Pattern Recognition and Artificial Intelligence, 16(8):1065-1086, 2002.

[24] J. Vreeken, M. van Leeuwen, and A. Siebes. Krimp: mining itemsets that compress. Data Min. Knowl. Discov., 23(1):169214, 2011. 